\title{
Energy Efficient Power Allocation for Collaborative Mobile Clouds with Information and Power Transfer
}

\author{
Zheng Chang*, Jie Gong ${ }^{\dagger}$, Tapani Ristaniemi*, Sheng Zhou ${ }^{\dagger}$, Zhisheng Niu ${ }^{\dagger}$ \\ * Department of Mathematical Information Technology, University of Jyväskylä, P.O.Box 35, FIN-40014 Jyväskylä, Finland \\ $\dagger$ Department of Electronic Engineering, Tsinghua National Laboratory for Information Science and Technology, \\ Tsinghua University, Beijing 100084, China \\ Email: \{zheng.chang, tapani.ristaniemi\}@jyu.fi, \{gongj13, sheng.zhou, niuzhs\}@tsinghua.edu.cn
}

\begin{abstract}
Prolonging the battery life of mobile terminals (MTs) is critical for mobile users, especially for the smartphone users to enjoy the high data rate services offered by the future wireless networks. A collaborative mobile cloud (CMC), which consists of several MTs offers one potential solution for reducing the energy consumption at the terminal side in the downlink. In addition, as RF signal can carry both information and energy simultaneously, the induced simultaneous wireless information and power transfer (SWIPT) is also capable of prolonging the battery of MTs. In this paper, the power allocation algorithm for CMC with SWIPT is formulated as a non-convex optimization problem which takes into account the baseband circuit power consumption, RF transmit and receiver power, harvested energy and the minimum required data rate. Accordingly, by exploiting the properties of nonlinear fractional programming, the formulated non-convex optimization problem, of which objective function is in fractional form, is transformed into an equivalent optimization problem having an objective function in subtractive form and is able to be solved in dual domain. Simulation results demonstrate that the proposed user scheduling and resource allocation algorithms can achieve significant energy saving performance.
\end{abstract}

Index Terms - content distribution; energy efficiency; content sharing; power allocation; collaborative mobile clouds; user cooperation.

\section{INTRODUCTION}

The battery capacity is improved at slow speed during last decades which creates the bottleneck in perpetuating the lifetime of the networks. Meanwhile, the rise of online services significantly increases the frequency of users' online activities. However, the demand for high data rate services is straining the current network as well as drawing battery of mobile devices much faster than before. Therefore, novel energy efficient approaches for network design are essential. Recently, as one emerging research area, energy harvesting (EH) technique received considerable attention due to its capability to realize self-maintenance elements in wireless networks. Meanwhile, conventional research on EH usually focused on the transmitter aspects, i.e. Base Stations (BS). Although, there are many $\mathrm{EH}$ resources, such as solar, wind and tide, they are usually either location- or weather-dependent. Thus, for the users in a closed/indoor space who can not access nature light or wind, EH becomes luxury and even impossible. As RF signal can carry both information and energy simultaneously, the induced simultaneous wireless information and power transfer (SWIPT) has gained much attention [1]. Through SWIPT, the receiver not only can decode data from transmitter, but also can "recycle" the transmit power and, thus, prolong the battery lifetime. In [1], the fundamental trade-offs between wireless information and power transfer were studied with the assumption that the receiver can simultaneously receive information and harvest energy from RF signal. In [2] and [3], authors proposed a receiver architecture which can split the received power into two power streams to facility the SWIPT. The work in [4] studied the outage probability of a cooperative EH relay network with multiple transmission pairs. In [5], the subcarrier and power allocation algorithms were proposed for multiuser OFDM system with SWIPT. Nonconvex optimization problem was formulated with objective to maximize the energy efficiency performance in term of bits/Joule.

Meanwhile, one of the proposed approaches to reduce energy consumption of mobile terminals (MTs) is to design cooperative content distribution architectures named Collaborative Mobile Clouds (CMCs) where MTs not only keep in touch with each other by messaging, but also can share some content and information cooperatively through Deviceto-Device (D2D) or Machine-to-Machine (M2M) communications [6]. A CMC consists of numbers of MTs that actively use two wireless interfaces: one to communicate with the BS over a Long-Range (LR) wireless technology and the other to cooperate with other MTs over a Short-Range (SR) communication link [7]. In a traditional service, each MT has to download the whole content on its own, which leads to significant energy consumption from MT batteries, especially if the LR data rates are low. Through the concept of CMC, several MTs can form a coalition and each MT can receive parts of required information data from BS, then exchange the received data with others [8]. In such case, one MT only needs to download parts of the data and consequently, the receiving time can be significantly reduced. Although information exchange over SR introduces new transmission overhead, the energy consumption can still be significantly decreased as the SR is generally more efficient in term of data rate [9].

In this work, we address the power allocation problem for the energy efficient design of CMC with hybrid information decoding (ID) and EH receivers. During the BS data delivery process, the dedicated receiver, denoted as information decod- 
ing MT (IMT) in the followings, will receive the assigned data and other MTs, denoted as energy harvesting MTs (EMTs) will harvest energy from the signal. After receiving from BS, IMT will transmit the data to other EMTs of the previous stage. The main contribution over existed works is three-fold.

- We first model the energy consumption of the overall transmission process when considering baseband circuit energy consumption, RF transmit and receive energy consumption and harvested energy.

- Then we focus on the algorithm design aspect and propose power allocation with objective of optimizing energy consumption performance of CMC.

- By using different optimization techniques and iterative algorithm design, we address the resulted non-convex programming problem. Simulation results are presented to illustrate the energy saving gain of the proposed scheme.

The rest of this paper is organized as follows. Section II describes the collaborative mobile cloud system model. In Section III, energy consumption models of ID and EH receivers, as well as CMC are presented. In Section IV, we formulate the optimization problem and introduce power allocation solution. We demonstrate the benefits of our proposed algorithm in Section V through simulation study and finally conclude this work in Section VI.

\section{System ModeL}

In our considered system, it is assumed that $K$ MTs which are geographically close to each other are interested in downloading the same content from a BS using a LR wireless technology (e.g., UMTS/HSPA, WiMAX, or LTE). One applicable area is the typical multicast multimedia service scenario. In this work, we consider the transmission frequency bandwidth on LR and SR is equal so that no extra bandwidth is invoked. The transmission process of CMC is depicted in Fig. 1 comparing with the conventional transmission, e.g., unicasting/multicasting. In the Fig. 1, the CMC consists of three MTs. In a conventional setup, the communication interface of a stand-alone MT has to remain active for the whole reception duration. This results in high energy consumption due to the required $\mathrm{RF}$ and baseband processing during data reception. In the $\mathrm{CMC}$, BS can distribute various and exclusive segments of data to different MTs, and MTs can then exchange/share the received parts with other members by utilizing the SR transmission among MTs. Thus, obviously the reception duration can be seriously reduced compared to the conventional transmission given that SR has better data rate than LR because of, e.g., its better channel condition. However, in order to share the received data, additional communication overheads are induced, such as transmit power of SR transmitter, transmit/receive durations. Consequently, the inherent energy efficiency performance calls for a careful algorithm design [10].

When considering the CMC with hybrid SWIFT MTs, the $\mathrm{CMC}$ members are able to receive power transfer when not being assigned data. Indeed, the SWIPT provides one potential

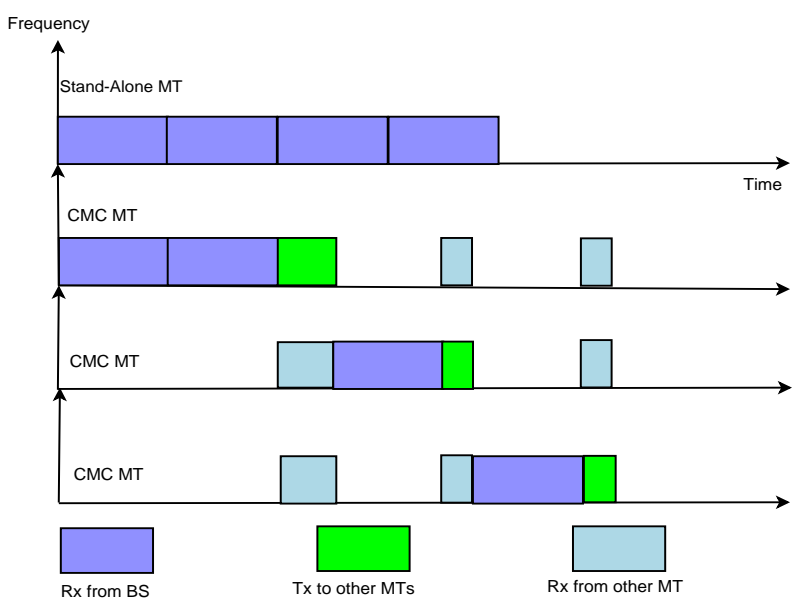

Figure 1. Transmission Procedure

solution for stimulating users to join and form the CMC. In the following, we will present the model for SWIPT receiver as well as formulate the resource allocation and user scheduling optimization problem in a CMC with hybrid receivers.

We assume quasi-static block fading channels and assume that the downlink channel state information can be accurately obtained by feedback from the receivers. All transceivers are equipped with single antenna. Let $x$ be the transmitted data from BS to receiver, then the received data can be modeled as

$$
y=\sqrt{P_{s, k}^{L_{t x}} L_{s, k} H_{s, k}} x_{s, k}+z_{s, k},
$$

where $x_{s, k}, P_{s, k}^{L_{t x}}$ and $H_{s, k}$ are the transmit data, transmit power and channel fading gain from BS to MT $k$, respectively. $L_{s, k}$ represents path loss from BS to MT $k . z_{s, k}$ is the additive Gaussian noise with zero mean and variance $\sigma_{z}^{2}$.

In order to focus on the resource allocation algorithm design and isolate it from the specific hardware implementation details, we do not assume a particular type of $\mathrm{EH}$ receiver. In this work, we focus on the receivers which consist of an energy harvesting unit and a conventional signal processing core unit for energy harvesting and information decoding. In addition, for the conventional signal processing, we separate receiver architecture into two parts, which are RF unit and baseband unit. In the following section, the energy consumption model of the considered system is presented.

\section{ENERGy CONSUMPTION MOdEL}

\section{A. Energy Harvesting Receiver}

In practice, the model of an $\mathrm{EH}$ receiver depends on its specific implementation. For example, electromagnetic induction and electromagnetic radiation are able to transfer wireless power, and the receiver is able to recycle the wireless power from radio signal [1]. Nevertheless, the associated hardware circuit in the receivers and the corresponding EH efficiency can be different. Besides, the signal used for decoding the modulated information cannot be used for harvesting energy due to hardware limitations [2]. In this work, we do not go to 
the details of particular type of EH receiver and the results of [3] are utilized.

We denote $P_{s, k}^{H}$ as the harvested power by EMT $k$ and the conversion efficiency $0<\vartheta_{k} \leq 1$. Then we have [3]

$$
P_{s, k}^{H}=\vartheta_{k} P_{s, k}^{L_{t x}} L_{s, k} H_{s, k},
$$

\section{B. Information Decoding Receiver}

For information receiver data rate on $\mathrm{LR} R_{s, k}^{L}$, the maximum achievable data rate from BS to MT $k$ is given as

$$
R_{s, k}^{L}=\log _{2}\left(1+\frac{P_{s, k}^{L_{t x}} L_{s, k} H_{s, k}}{\sigma_{z}^{2}}\right),
$$

In the CMC, the IMT $k$ needs to multicast its received data to other CMC members, so the data rate on the SR link can be expressed as,

$$
R_{k}^{S}=\log _{2}\left(1+\frac{P_{k}^{S_{t x}} L_{k} H_{k}}{\sigma_{z}^{2}}\right),
$$

where $P_{k}^{S_{t x}}$ is the multicast transmit power of IMT $k, L_{k}$ and $H_{k}$ are the path loss and channel gain from $k$ to the MT with worst channel condition, respectively. We also assume that the noises on LR and SR are of same kind.

\section{Tx and Rx Energy Consumption of CMC}

As we know, the energy consumption can be modeled as a linear function containing the power consumption and the time duration. Therefore, the energy consumption for receiving data size $S_{T}$ from BS can be expressed as

$$
\begin{aligned}
E_{s, k}^{L_{r x}} & =\left(P_{s, k}^{L_{r x}}+P_{E}\right) T_{s, k}^{L_{r x}}=\frac{\left(P_{s, k}^{L_{r x}}+P_{E}\right) S_{T}}{R_{s, k}^{L}} \\
& =\frac{\left(P^{r x}+P_{E}\right) S_{T}}{R_{s, k}^{L}}
\end{aligned}
$$

where $P_{s, k}^{L_{r x}}$ is the RF power consumption of $k$ for receiving from BS and $P_{E}$ is the electronic circuit power consumption of baseband. $T_{s, k}^{L_{r x}}=\frac{S_{T}}{R_{s, k}^{L}}$ is the required time for receiving data $S_{T}$ on LR subchannel $i$. Further we can assume the receive RF power consumption are the same for both LR and SR links, and equals to $P_{r x}$. After receiving from the BS, MT $k$ is going to transmit its received data to other required MTs. There are two conventional ways to deliver data inside CMC, which are unicasting and multicasting. We have discussed the energy efficiency of using both two schemes in [10]. So in this work, we only invoke multicasting as the transmission strategy inside CMC.

If multicasting is used, an IMT only needs to broadcast its data to other MTs in CMC once with the data rate that can reach the MT with worst channel condition. Thus with the assumption of transmitting time $T_{k}^{S_{t x}}$, the transmit energy consumption of IMT is given as

$$
E_{k}^{S_{t x}}=\left(P_{k}^{S_{t x}}+P_{E}\right) T_{k}^{S_{t x}}=\frac{\left(P_{k}^{S_{t x}}+P_{E}\right) S_{T}}{R_{k}^{S}} .
$$

Therefore, the total energy consumption of CMC when using MT $k$ as the IMT can be expressed as

$$
E_{k}=E_{s, k}^{L_{r x}}+E_{k}^{S_{t x}}+\sum_{n, n \neq k}^{K} E_{n}^{S_{r x}} .
$$

$E_{k}$ is the energy consumption of IMT $k$ for receiving from BS and multicasting its received data. $E_{n}^{S_{r x}}$ is the energy consumption of each EMT when receiving from IMT, and it can be expressed as

$$
\begin{aligned}
E_{n}^{S_{r x}} & =\left(P_{n}^{S_{r x}}+P_{E}\right) T_{k}^{S_{r x}}=\frac{\left(P_{n}^{S_{r x}}+P_{E}\right) S_{T}}{R_{k}^{S}} \\
& =\frac{\left(P_{r x}+P_{E}\right) S_{T}}{R_{k}^{S}} .
\end{aligned}
$$

\section{Energy Consumption of Base Station}

In the previous part, we presented the energy consumption of MTs in a CMC during one data segment assignment. Obviously, the energy consumption of the BS is given as,

$$
E_{s}^{L_{t x}}=\left(P_{s, k}^{L_{t x}}+P_{B}\right) T_{s, k}^{L_{r x}}=\frac{\left(P_{s, k}^{L_{t x}}+P_{B}\right) S_{T}}{R_{s, k}^{L}},
$$

where $P_{B}$ is the BS baseband operating power consumption.

\section{Power Allocation Problem}

\section{A. Problem Formulation}

In the previous section, we presented the energy consumption model of the CMC with hybrid ID-EH receivers. In this section, the considered problem will be formulated with objective to minimize the energy consumption during each transmission time of sending data $S_{T}$.

In order to minimize the energy consumption of a CMC, at first one MT inside CMC will be selected as the IMT and other MTs will be considered as EH receivers. Then the BS transmit the data to IMT with data rate $R_{s, k}^{L}$. After that, IMT will act as a relay and forwards the received data to other MTs with multicast data rate $R_{k}^{S}$.

To this end, taken harvested energy into consideration, for each data segment transmission, we can formulate the optimization objective as,

$$
\mathcal{E}(\mathbf{P})=E_{s}^{L_{t x}}+E_{k}-Q_{s, n},
$$

where $K$ is the total number of MTs inside CMC and $\mathbf{P}=\left\{P_{s, k}^{L_{t x}}, P_{k}^{S_{t x}}\right\}$ is the power allocation policy. $Q_{s, n}$ is the harvest energy and obversely $Q_{s, n}=P_{s, n}^{H} S_{T} / R_{s, k}^{L}$. Note that mathematically $\mathcal{E}(\mathbf{P})$ can take the negative value. However, $\mathcal{E}(\mathbf{P})$ always holds for practical system. Therefore, the power allocation optimization problem can be formulated as 


$$
\min _{\mathbf{P}} \mathcal{E}(\mathbf{P})
$$

s.t.

$$
\begin{aligned}
& C 1: R_{s, k}^{L}>R_{\text {min }}, \\
& C 2: R_{k}^{S}>R_{\text {min }}, \\
& C 3: P_{s, k}^{L_{t x}} \leq P_{s, \text { max }}, \\
& C 4: P_{k}^{S_{t x}} \leq P_{k, \text { max }},
\end{aligned}
$$

Here, the optimization problem (11) is formulated with several constraints. $R_{\min }$ in $\mathrm{C} 1$ and $\mathrm{C} 2$ is the required Quality of Service (QoS) data rate and the data rates of both LR and SR should be higher than $R_{\text {min }}$. C3 ensures that the power allocation of BS and IMT should not be higher than the maximum allowed transmit power.

It is worth noticing that (11) with (12) is with a non-convex structure. In general, there is no standard approach for solving such a non-convex optimization problem. In the extreme case, an exhaustive search or branch-and-bound method is needed to obtain the global optimal solution. In order to make the problem tractable, we transform the objective function and approximate the transformed objective function in order to simplify the problem.

\section{B. Proposed Power Allocation Scheme}

One may find that the objective function (11) is quasiconvex function w.r.t. to the power allocation policy. Therefore, as a result, an unique global optimal solution exists and the optimal point can be obtained by using the bisection method [11]. We can also apply the nonlinear fractional programming method [12] to solve the formulated power allocation problem.

1) Problem Transformation: One may notice that obtaining power allocation policy involves solving $\mathcal{E}(\mathbf{P})$, which can be expressed as in (13). To simplify the expression, we arrive at

$$
\mathcal{E}(\mathbf{P})=\mathcal{E}_{L R}\left(P_{s, k}^{L_{t x}}\right)+\mathcal{E}_{S R}\left(P_{k}^{S_{t x}}\right),
$$

where $\mathcal{E}_{L R}\left(P_{s, k}^{L_{t x}}\right)=\frac{U_{1}\left(P_{s, k}^{L_{t x}}\right)}{R_{1}\left(P_{s, k}^{L_{t x}}\right)}$ and $\mathcal{E}_{S R}\left(P_{k}^{S_{t x}}\right)=\frac{U_{2}\left(P_{k}^{S_{t x}}\right)}{R_{2}\left(P_{k}^{S_{t x}}\right)}$. From (14), one can observe that the power allocation schemes for BS and IMT are separated. In other word, we can obtain optimal power allocation by addressing $\mathcal{E}_{L R}\left(P_{s_{t} k}^{L_{t x}}\right)$ and $\mathcal{E}_{S R}\left(P_{k}^{S_{t x}}\right)$ individually. We can see that both $\mathcal{E}_{L R}\left(P_{s, k}^{L_{t x}}\right)$ and $\mathcal{E}_{S R}\left(P_{k}^{S_{t x}}\right)$ are quasi-convex functions w.r.t. power allocation variables, respectively. For the sake of presentation simplicity, we introduce a method for solving $\mathcal{E}_{L R}\left(P_{s, k}^{L_{t x}}\right)$ which is derived from nonlinear fractional programming [12].

Considering the global optimal solution $q_{L R}^{*}$, which can be expressed as

$$
q_{L R}^{*}=\mathcal{E}_{L R}\left(P_{s, k}^{L *}\right)=\min _{P_{s, k}^{L_{t x}}} \frac{U_{1}\left(P_{s, k}^{L_{t x}}\right)}{R_{1}\left(P_{s, k}^{L_{t x}}\right)} .
$$

Therefore, the optimal energy efficiency $q_{L R}^{*}$ can be obtained iff [12]

$$
\min _{P_{s, k}^{L_{t x}}} U_{1}\left(P_{s, k}^{L_{t x}}\right)-q_{L R}^{*} R_{1}\left(P_{s, k}^{L_{t x}}\right)=0,
$$

which gives a necessary and sufficient condition w.r.t. optimal power allocation. Particularly, for the considered optimization problem with an objective function in fractional form, there exists an equivalent optimization problem with an objective function in subtractive form, i.e., $U_{1}\left(P_{s, k}^{L_{t x}}\right)-q_{L R}^{*} R_{1}\left(P_{s, k}^{L_{t x}}\right)$, and both formulations result in the same power allocations. To achieve the optimal $q_{L R}^{*}$, the iterative algorithm with guaranteed convergence in [12] can be applied. The iterative algorithm is given in Alg. 1.

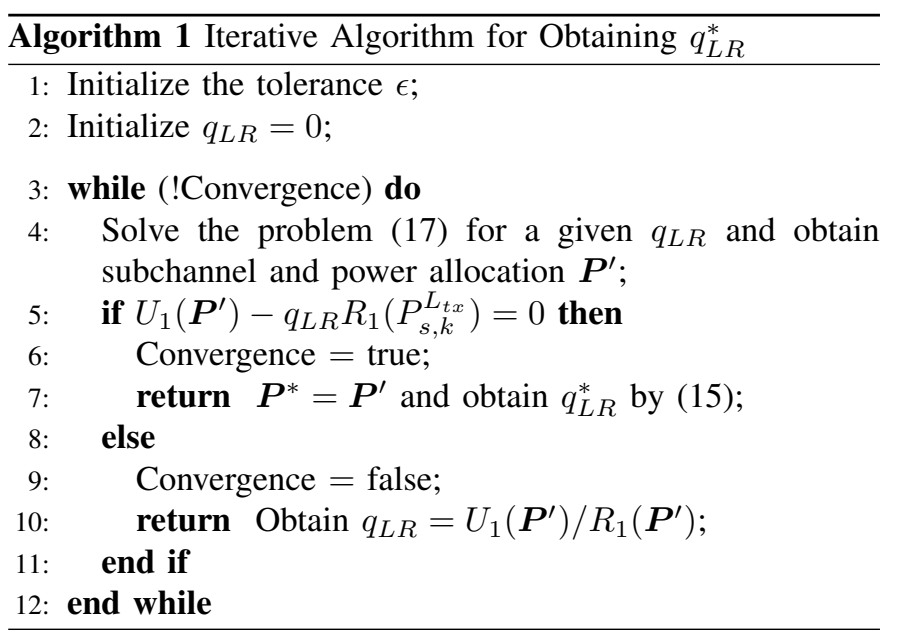

During the iteration, in order to achieve $q_{L R}^{*}$, we need to address the following problem with $q_{L R}$ which is given as

$$
\min _{P_{s, k}^{L_{t x}}} U_{1}\left(P_{s, k}^{L_{t x}}\right)-q_{L R} R_{1}\left(P_{s, k}^{L_{t x}}\right),
$$

s.t.

$$
\begin{aligned}
& R_{s, k}^{L}>R_{\text {min }}, \\
& P_{s, k}^{L_{t x}} \leq P_{s, \text { max }},
\end{aligned}
$$

To this end, the transformed problem is able to be solved by exploiting the convexity of the problem. It can been seen that strong duality holds for the transformed problem, then solving the dual problem is equivalent to solving the primal problem.

2) Dual Problem Formulation and Decomposition: In this part, we solve the power allocation optimization problem of LR link by solving its dual for a given value of $q_{L R}$. The Lagrangian function of the primal problem (16) can be given as,

$$
\begin{aligned}
\mathcal{L}(\mathbf{P}, \lambda, \theta)= & U_{1}\left(P_{s, k}^{L_{t x}}\right)-q_{L R} R_{1}\left(P_{s, k}^{L_{t x}}\right)-\lambda\left(R_{s, k}^{L}-R_{\min }\right) \\
& -\mu\left(P_{s, k}^{L_{t x}}-P_{s, \max }\right),
\end{aligned}
$$

where $\lambda, \mu$ are the lagrange multipliers associated with different constraints. Therefore, the dual problem is 


$$
\mathcal{E}(\mathbf{P})=\frac{\overbrace{S_{T}\left(P_{s, k}^{L_{t x}}+P_{B}+P_{r x}+P_{E}-\vartheta_{n} P_{s, k}^{L_{t x}} L_{s, n} H_{s, n}\right)}^{U_{1}\left(P_{s, k}^{L_{t x}}\right)}}{\underbrace{R_{s, k}^{L}}_{R_{1}\left(P_{s, k}^{L_{t x}}\right)}}+\frac{\overbrace{S_{T}\left(P_{k}^{S_{t x}}+(K-1) P_{r x}+K P_{E}\right)}^{U_{2}\left(P_{k}^{S_{t x}}\right)}}{\underbrace{R_{k}^{S}}_{R_{2}\left(P_{k}^{S_{t x}}\right)}}
$$

$$
\max _{\lambda, \mu} \min _{\mathbf{P}} \mathcal{L}(\mathbf{P}, \lambda, \mu) .
$$

By using Lagrange dual decomposition, the dual problem (20) can be decomposed into two layers, minimization of (19) which is the inner problem and maximization of (20) which is the outer problem. The dual problem can be solved by addressing both problems iteratively, where in each iteration, the optimal power allocation and subchannel allocation can be obtained by using the KKT conditions for a fixed set of Lagrange multipliers, and the outer problem is solved using the (sub)gradient method [14].

Using convex optimization techniques and applying the KKT conditions, the closed-form optimal power allocation for user $k$ for a given $q_{L R}$ can be obtained, i.e. $\frac{\partial \mathcal{L}(\mathbf{P}, \lambda, \mu)}{\partial P_{s, k x}^{L_{t}}}=0$.

In order to find the optimal solutions of the lagrange multipliers of outer problem of (20) for the given resource allocation policies, (sub)gradient algorithm can be used as a dual function which is always convex and differentiable.

To summarize the iterative algorithm between inner and master problems, the multiplier updates can be interpreted as the pricing adjustment rule of the demand and supply model [16]. Specifically, the Lagrange multipliers can be interpreted as a set of shadow prices for utilizing the resources. If the demand of the resource exceeds the supply, then the gradient method will raise the shadow prices via adjusting the Lagrange multipliers in the next iteration; otherwise, it will reduce the shadow prices until some resources can afford them [5]. By combining the gradient updates, power allocation problem is able to be addressed through transformed problem in (17).

We have presented the scheme on how to address the minimization of $\mathcal{E}_{L R}\left(P_{s, k}^{L_{t x}}\right)$. Same procedure can be applied to obtain the optimal power allocation solution of minimizing $\mathcal{E}_{S R}\left(P_{k}^{S_{t x}}\right)$. Then we are able to obtain the solution set of (11).

\section{Simulation Results}

\section{A. Simulation Setting}

The channel coefficients are assumed to be constant during a complete data transmission, and can vary one to another independently. For the SR transmission link, the path loss follows the IEEE $802.11 \mathrm{ac}$ standards with $5 \mathrm{GHz}$ central frequency. The noise variance is assumed 1 for simplicity. Although the baseband power $P_{E}$ and $P_{B}$ are not constant in general and their values depend on the features of circuit design, in this work it is out of the scope and we assume they are fixed

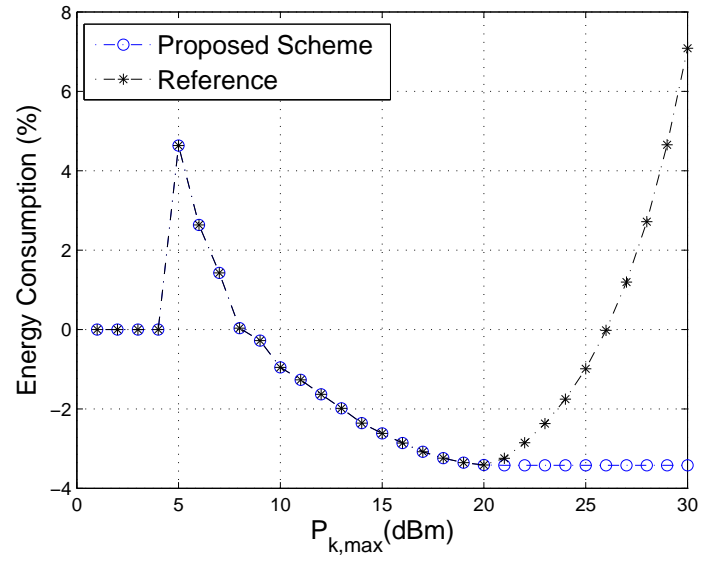

Figure 2. Overall System Energy Consumption
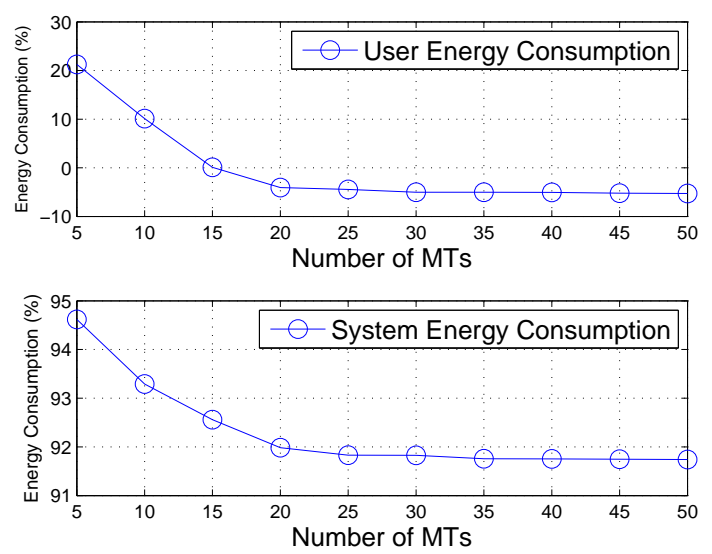

Figure 3. User Energy Consumption

according to parameters in [8]. To illustrate the energy saving performance, we compare our resource allocation scheme with pure multicasting transmission, that is, the reference energy consumption is the one when BS use multicasting to deliver all data to every MT. The energy consumption ratio (EC) is obtained by normalized the presented system with the conventional multicasting scheme. In the user location setup, we consider BS is about $500 \mathrm{~m}$ from MTs and MTs randomly are located in a $50 \times 50 \mathrm{~m}^{2}$ square. 


\section{B. Performance Evaluations}

Fig. 2 depicts the energy consumption of 30 MTs versus the $P_{k, \max }$. In addition to use multicasting as the reference, we also compare our proposed scheme with one reference scheme that is obtained by modifying the objective function (11) to the throughput maximization (TP). It can be seen that for $P_{k, \max }<5 \mathrm{dBm}, E C=0$ since the optimization problem is infeasible due to an insufficient power transmission to satisfy the constraints on $R_{\min }$. For a larger $P_{k, \max }$, the energy consumption of the proposed algorithm first decreases with increasing $P_{k, \max }$ and then approaches a constant due to a higher transmit power allowance gets saturated. It can be found that before saturation, the proposed scheme can achieve throughput maximization as well. It can be also noticed that the energy consumption of the reference scheme increases dramatically in the high transmit power regime. This is because such scheme employs a large transmit power for throughput maximization which causes low energy efficiency. The negative value on the y-axis of Fig. 2 implies that the harvested energy at MTs is higher that the consumed energy, which means that the SWIPT is appreciated for the MTs who are facing energy consumption problems.

In Fig. 3, we vary the number of MTs inside a CMC to plot the energy saving performance. For example, in the upper one of Fig. 3, the performance of MTs' energy consumption is shown which exclude the energy consumption of BS. WE can see that by our proposed scheme for CMC with hybrid receivers, the energy consumption can be significantly reduced up to $90 \%$ when there are 10 MTs in a CMC. Such energy saving benefit came from the fact that the best MT who can minimize the energy consumption during reception process can be selected and then forward the data to others with best data rate. The EH feature of MTs can also improve the energy saving performance.

In the lower figure of Fig. 3, the overall system energy consumption is presented taking into consideration of both energy consumptions of BS and all MTs. DMT to the fact that $\mathrm{BS}$ has relative higher energy consumption comparing with MTs, we can see that the overall energy consumption reduction is only up to $9 \%$ when there are 50 MTs forming the CMC. Although the energy saving is not that dramatic comparing with the one when only considering MTs, our proposed scheme can still offer an option to reduce the energy consumption of conventional multicasting transmission.

\section{CONCLUSION}

In this paper we have investigated the problem of power allocation for collaborative mobile clouds with hybrid receivers. We have proposed an power allocation algorithm which can noticeably obtain energy efficiency performance. The formulated optimization problem was addressed by using nonlinear fractional programming and dual decomposition. Through simulation study, it manifested that by designing a proper power allocation scheme, it is possible to achieve a noticeable gain in the energy consumption for the considered system during receiving process.

\section{REFERENCES}

[1] P. Grover and A. Sahai, "Shannon meets Tesla: Wireless information and power transfer," in Proc. of IEEE International Symposium on Information Theory, Austin, TX, June 2010.

[2] R. Zhang and C. K. Ho, "MIMO broadcasting for simultaneous wireless information and power transfer," IEEE Transaction on Wireless Communications, Vol. 12, No. 5, pp. 1989-2001, May 2013.

[3] X. Zhou, R. Zhang and C-K Ho, "Wireless Information and Power Transfer: Architecture Design and Rate-Energy Tradeoff," in Proc. of IEEE Global Communications Conference (GLOBECOM), pp. 39823987, Anaheim, CA, Dec. 2012.

[4] Z. Ding and H. V. Poor, "Cooperative Energy Harvesting Networks with Spatially Random Users," IEEE Signal Processing Letters, Vol. 20, No. 12, pp. 1211-1214, Dec. 2013.

[5] D. W. K. Ng, E. Lo, and R. Schober, "Wireless Information and Power Transfer: Energy Efficiency Optimization in OFDMA Systems," IEEE Transactions on Wireless Communications, Vol. 12, No. 12, pp. 63526370, Dec. 2013.

[6] M. V. Pederson and F. H. P. Fitzek, "Mobile Clouds: The New Content Sharing Platform," Proceeding of IEEE, Vol. 100, pp. 1400-1403, 2012.

[7] L. Al-Kanj, Z. Dawy, and E. Yaacoub, "Energy-Aware Cooperative Content Distribution over Wireless Networks: Design Alternatives and Implementation Aspects," IEEE Communications Surveys and Tutorials, Vol. 15, No. 4, pp. 1736-1760, Dec. 2013.

[8] Z. Chang and T. Ristaniemi, "Energy Efficiency of Collaborative OFDMA Mobile Cluster," in Proc. of 10th IEEE Consumer Communications and Networking Conference (CCNC'13), Las Vegas, NV, Jan. 2013.

[9] Z. Chang and T. Ristaniemi, "Collaborative Mobile Clusters: An EnergyEfficient Emerging Paradigm". book chapter in "Broadband Wireless Access Networks for 4G: Theory, Application, and Experimentation", IGI Global, Dec. 2013.

[10] Z. Chang and T. Ristaniemi, "Efficient Use of Multicast and Unicast in Collaborative OFDMA Mobile Cluster," in Proc. of IEEE 77th Vehicular Technology Conference (VTC'13-spring), Dresden, Germany, Jun. 2013.

[11] S. Boyd, and L. Vandenberghe, Convex Optimization, Cambridge UK: Cambridge University Press, 2004.

[12] W. Dinkelbach, "On Nonlinear Fractional Programming," Management Science, Vol. 13, pp. 492íC498, Mar. 1967.

[13] W. Yu and R. Lui, "Dual Methods for Nonconvex Spectrum Optimization in Multicarrier System," IEEE Transaction on Communications, Vol. 54, No. 7, pp. 1310-1322, Jul. 2006.

[14] S. Boyd, and A. Mutapcic, Lecture Notes for EE364b , Standford University , 2006-2007.

[15] Z. Chang and T. Ristaniemi, "Asymmetric Radio Resource Allocation Scheme for OFDMA Wireless Networks with Collaborative Relays," Wireless Networks, Vol. 19, No. 5, pp. 619-627, DOI: 10.1007/s11276012-0490-8.

[16] D. W. K. Ng, E. Lo, and R. Schober, "Energy-Efficient Resource Allocation in OFDMA Systems with Large Numbers of Base Station Antennas," IEEE Transactions on Wireless Communications, Vol. 11, No. 9, pp. 32921́C3304, Sep. 2012. 